\title{
Aero Thermodynamic Structural Topology Optimization Of Hypersonic Re-entry Capsule By Using CFD
}

\author{
P.Harish ${ }^{1}$, A.S.Shabaz ${ }^{2}$ \\ ${ }^{I}$ (Asst. professor, Mechanical Engineering, Sri Venkateswara College of Engineering and Technology, \\ Autonomous, India) \\ ${ }^{2}$ (U.G scholar, Mechanical Engineering, Sri Venkateswara College of Engineering and Technology, \\ Autonomous, India)
}

\begin{abstract}
A re-entry capsule is the portion of a spacecraft which returns to earth following a space flight. In present paper, a fluent analysis is performed on two distinctive models of capsule and temperature contours are outlined. Firstly, the aft end formed in a sharp (slender body) shape forms a shock wave that touches the capsule and high temperatures are formed on the body, Whereas, the aft end is formed in a rounded (blunt body) shape, as this forms a shock wave that doesn't touch the capsule, and the heat is diverged away rather than melting the vehicle body and low temperatures are seen on the body. The capsule model was created in Solid Works, meshing and general post processing was carried out in Ansys fluent 14.0
\end{abstract}

Keywords: aerothermodynamics, atmospheric entry, domain, hypersonic re-entry vehicle, topology

\section{Introduction}

During the conceptual design stage of re-entry vehicles, the design parameters are varied to gain knowledge of their effect on the vehicle's overall performance. One of the defining aspects of an entry vehicle is its shape and geometry, as it will largely define the aero thermodynamic characteristics of the vehicle. The continuous modeling of the vehicle shape, and resulting entry performance as a function of this shape, are useful for analyzing the influence of shape variations. The goal of the work described in this paper is to set up a vehicle topology optimization that can be used during the conceptual design phase. To this end, it is required that the evaluation and optimization procedures are relatively fast, allowing many different runs to be performed for various types of shapes. In this paper, two vehicle parameterizations are considered, both of which allow continuous shape variation. First, an entry capsule of blunt shape is proposed. Second, an entry capsule of slender shape is considered. In aerodynamic design and optimization problems, a design's performance can be estimated using numerical models based on computational fluid dynamics $(C F D)$ techniques.

\section{Geometry model}

The 2D geometries were drafted by using Solid Works software as shown in figure $2.1 \& 2.2$

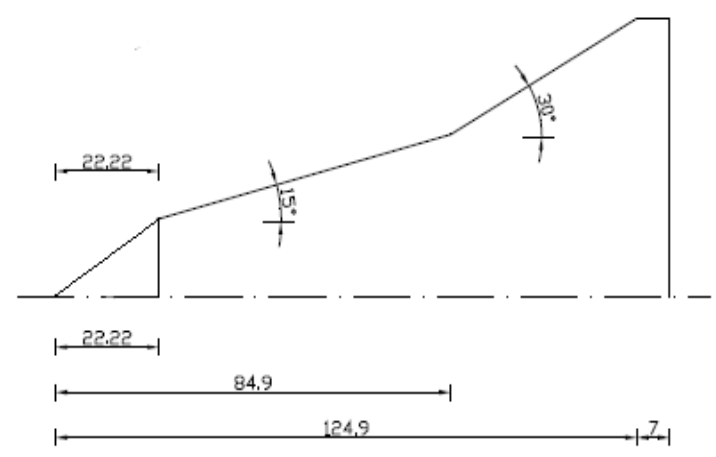

Fig.2.1 2d geometry of slender body

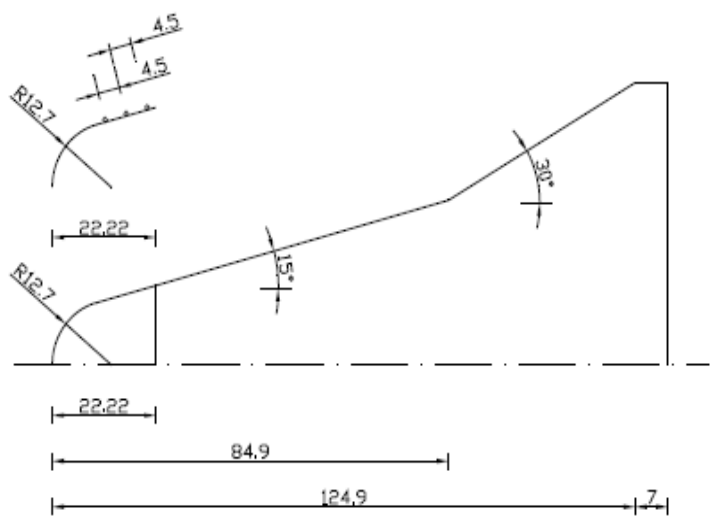

Fig.2.2 2d geometry of blunt body

\subsection{Meshing}

Meshing was made very fine at the edges and coarse as it goes away from the body. At the edge surfaces inflation with 5 layers is used. The geometries were imported to Ansys fluent 14.0 and mesh was generated as shown in the figure $2.1 .1 \& 2.1 .2$ 


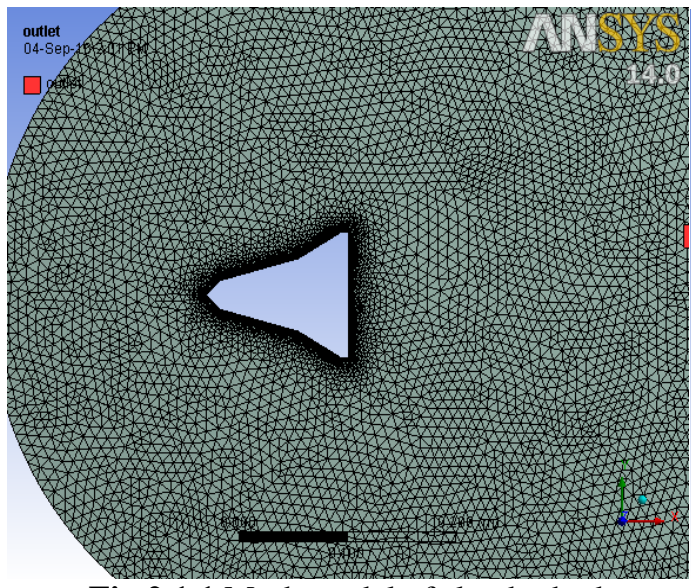

Fig.2.1.1 Mesh model of slender body

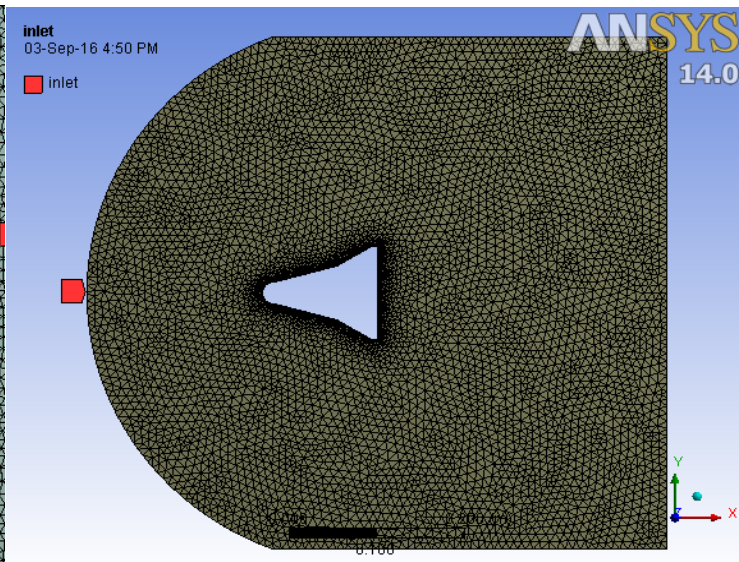

Fig.2.1.2 Mesh model of blunt body

The domain dimensions are $1 l$ from major length of the model at the face and $1.5 l$ times behind the geometry and $1.25 \mathrm{l}$ on the top and bottom of the geometry model.

\subsection{Boundary conditions}

Pressure inlet condition was set to the inlet of the meshed model where inlet pressure is specified and pressure far field was set to the outlet and top and bottom of model and body was set to the wall boundary condition. The flow conditions used in fluent setup are as show below

\begin{tabular}{|c|c|}
\hline Inlet & 50 bar \\
\hline Outlet & 0.0 .0022025 bar \\
\hline Body & Wall \\
\hline Temperature & $773 \mathrm{k}$ \\
\hline Mach No & 9.1 \\
\hline
\end{tabular}

The CFD analysis was conducted for density based implicit formulation 2D Flow, the compressible flow is coupled to the energy equation for the flow simulation using Spalart -Allmaras Model. Same boundary conditions were applied to both the geometries and iterated in setup module.

\section{Results and discussion}

The results of fluent setup are indicated in the following session. Temperature profiles of blunt shape and slender shape vehicle are also shown.

\subsection{Temperature contour of slender shape vehicle}

The temperature contour of blunt body reveals that a static temperature of $1125 \mathrm{k}$ is observed on tip of the capsule which is due to separation of shock wave on the slender body
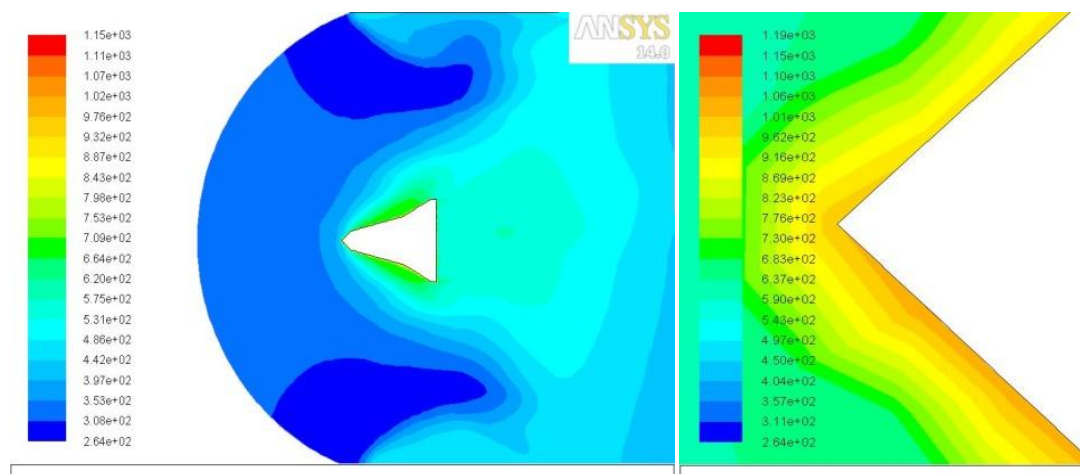

Contours of Static Temperature $(k)$

ANSYS FLUENT 14.0 (2d, dp, dubns 26 imp, 201

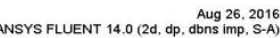

Fig.3.1.1 Temperature profile of slender body at 50 bars The enlarged profile of the slender body at the tip is shown in figure 3.1.2 


\subsection{Temperature contour of blunt shape vehicle}

The temperature contour of blunt body reveals that a static temperature of $815 \mathrm{k}$ is observed on nose of the capsule which is due to the subsonic flow behind bow shock wave of the body

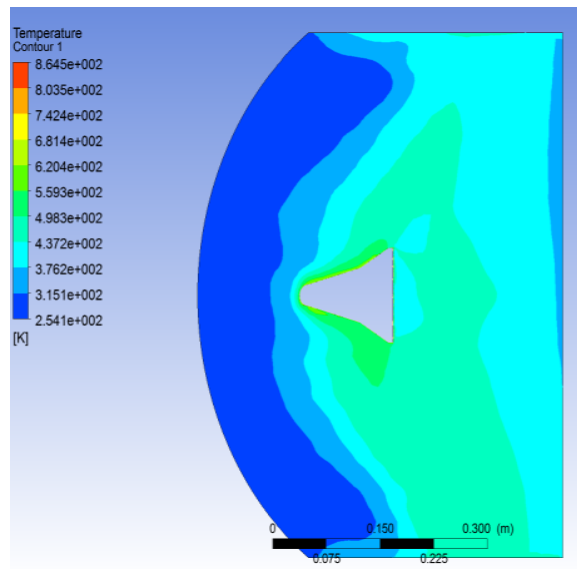

Fig 3.2.1 Temperature profile of blunt body at 50 bars

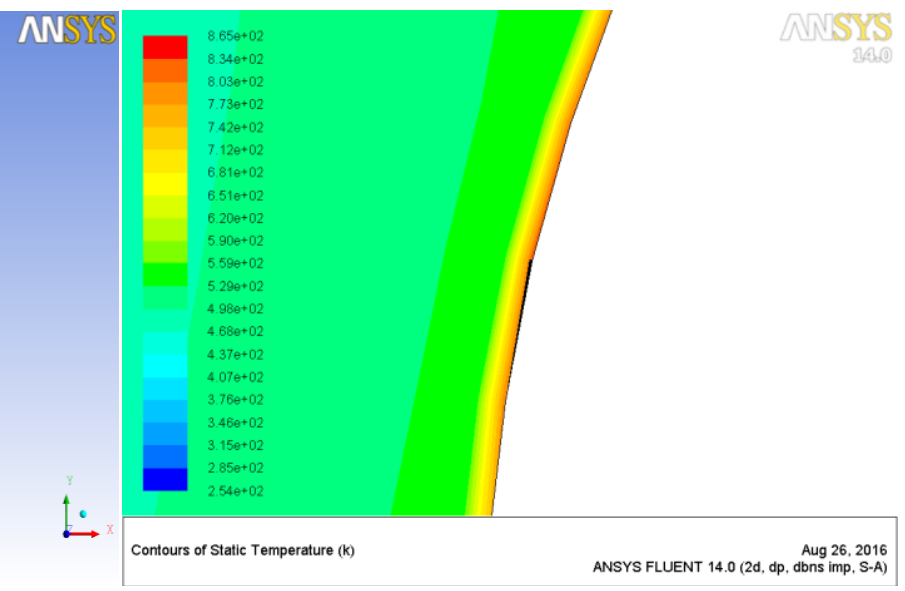

Fig.3.2.2 Enlarged View of Temperature profile

The enlarged view of the blunt body at the node edge is shown in figure 3.2.2. It gives a clear idea of temperatures at nose edge of the capsule.

\subsection{Pressure profiles of blunt and slender body}

Contours of static pressure (pa) representing the pressure at blunt nose region is high which will also create drag. But as far as aeroheating is concerned blunt shape is economic. Figure 3.3.1 shows the pressure profiles.

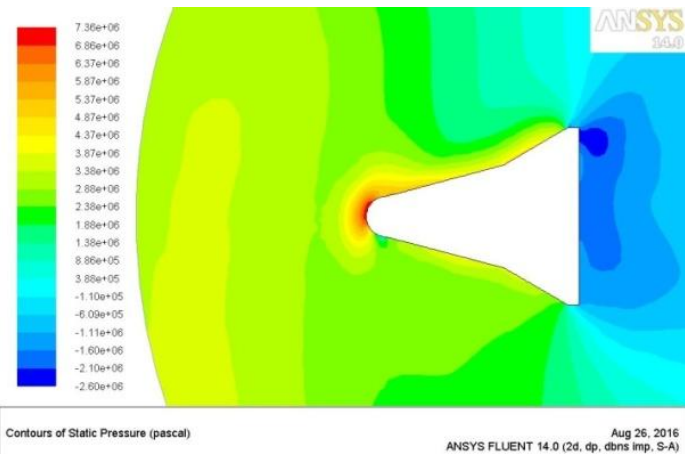

Fig.3.3.1 Contour of static pressure (blunt body)

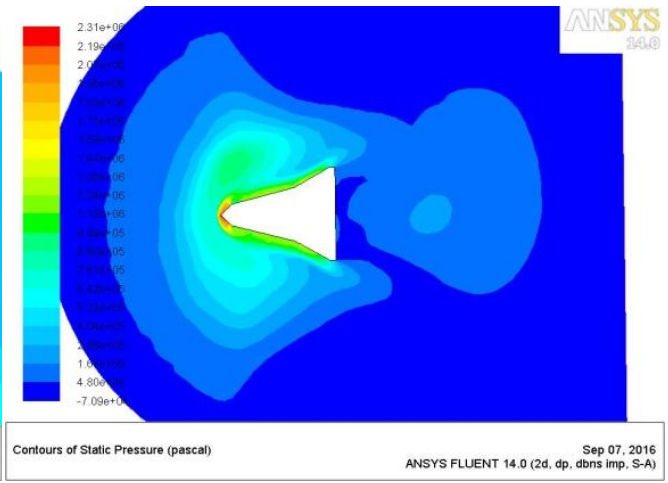

Fig.3.3.2 Contour of static pressure (slender body)

\subsection{Plots of temperature vs. Length of the vehicle}

Temperature of the vehicle at each node i.e along length of the vehicle is shown in figure 3.4.1 \& 3.4.2. It clearly indicates the temperatures at every node if the vehicle.

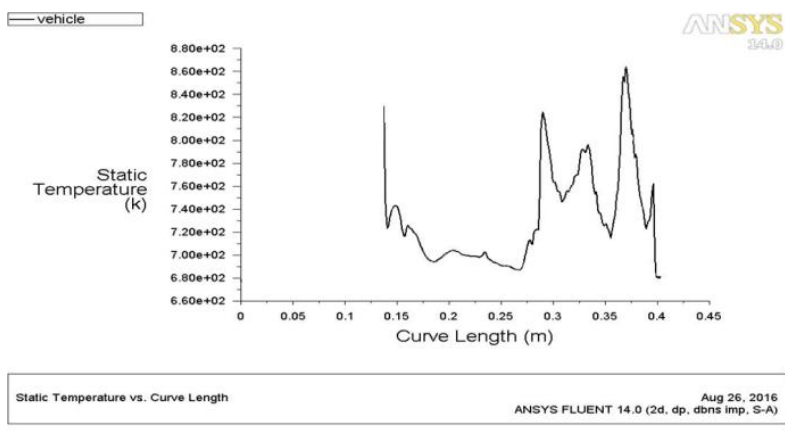

Fig. 3.4.1 Plot of blunt vehicle

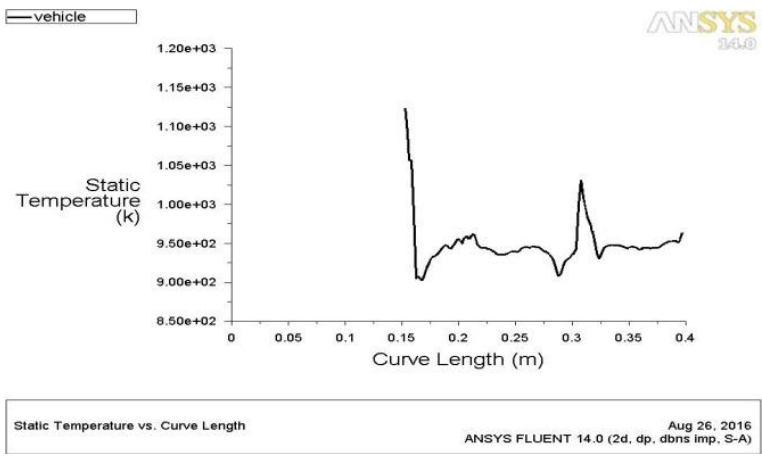

Fig. 3.4.2 Plot of slender vehicle 


\section{Conclusion}

In spite of the decades of research into hypersonic flow; there are still many challenges for analyzing and designing high-speed vehicles. While simulating the Blunt \& Slender hypersonic vehicle, the temperature of the Blunt vehicle reduces by $26.2 \%$ with respect to slender body at the nose due to bow shock wave and also reduction in the pressure of the vehicle so that there is less possibility or more time to cause explosion.

\section{References}

[1]. Ferry F.J. Schrijer and Fulvio Scarano and Bas W. van Oudheusden conducted on experiment on Hypersonic Boundary Layer Separation and Reattachment on a Blunted Cone-Flare using Quantitative Infrared Thermography 2003.

[2]. P.Harish, K. Rajagopal, Aerothermodynamic Design optimization of a hypersonic winged re-entry vehicle using CFD, International Journal Of Engineering and Technology, Volume 3-Issue 7

[3]. John D Anderson Computational fluid dynamics (International Editions 1995)

[4]. M.Y.M. Ahmeda N. Q investigated Recent advances in the aerothermodynamics of spiked hypersonic vehicles 2011

[5]. Zonglin Jiang and Yunfeng Liu and Guilai Hanwas conducted on In order to achieve efficient wave drag reduction under non-zero attack angles and avoid the severe aerodynamic heating, a new concept of the Non-ablative Thermal Protection System (NATPS) for hypersonic vehicles. 2011.

[6]. N.Sreenivasa Babu. Dr. K. Jayathirtha Raowas studied Aerodynamic drag and heating are the crucial in the thermal stability of hypersonic vehicles at various speeds.

[7]. D. Dirkx, E. MooijDelft University of Technology, Faculty of Aerospace Engineering, Section of Aerodynamics and Space Missions, Kluyverweg

[8]. D. Kinney, Aerodynamic Shape Optimization of Hypersonic Vehicles, 44th AIAA Aerospace Sciences Meeting and Exhibit, No. AIAA 2006- 239, 2006. 